Voix et Images

\title{
De plain-pied dans l'écriture. Entretien avec André Brochu
}

\section{François Dumont}

Volume 20, numéro 3 (60), printemps 1995

André Brochu

URI : https://id.erudit.org/iderudit/201186ar

DOI : https://doi.org/10.7202/201186ar

Aller au sommaire du numéro

\section{Éditeur(s)}

Université du Québec à Montréal

\section{ISSN}

0318-9201 (imprimé)

1705-933X (numérique)

Découvrir la revue

\section{Citer ce document}

Dumont, F. (1995). De plain-pied dans l'écriture. Entretien avec André Brochu. Voix et Images, 20(3), 519-529. https://doi.org/10.7202/201186ar d'utilisation que vous pouvez consulter en ligne.

https://apropos.erudit.org/fr/usagers/politique-dutilisation/ 


\section{De plain-pied dans l'écriture. Entretien avec André Brochu}

\section{François Dumont, Université du Québec à Trois-Rivières}

V. et I. : André Brochu, il me semble qu'un changement de proportion assez net s'est opéré, depuis quelques années, entre votre travail de critique et votre travail de création. Mais est-il vraiment pertinent, selon vous, de distinguer votre travail de critique de votre travail d'écrivain?

A. B. : Je me suis orienté vers l'écriture dès mon tout jeune âge. Mais il m'a fallu beaucoup de temps avant d'arriver à une pratique de l'écriture satisfaisante. La critique littéraire a donc pris le relais pendant de nombreuses années.

V. et I. : Vous perceviez les choses comme cela dès le début?

A. B. : J'ai longtemps voulu voir dans la critique littéraire un équivalent de l'écriture, parce que je n'étais pas sûr de pouvoir réaliser un jour le passage que j'espérais. J'ai donc voulu que la critique littéraire soit plus qu'un simple travail intellectuel, qu'elle soit une activité d'écriture au sens plein du terme. Mais en même temps, malgré ce que j'ai pu déclarer à l'époque sur le sujet, je n'étais jamais tout à fait convaincu que la critique littéraire puisse remplacer l'écriture telle que j'aurais souhaité la pratiquer, c'est-à-dire l'écriture poétique et narrative. La critique littéraire m'a certainement permis d'entretenir le rêve de l'écriture, et cela, de plusieurs façons. Elle me mettait en contact avec des textes qui représentaient pour moi l'idéal de l'écriture, chacun à sa manière. Et puis, même si la critique littéraire est affaire de savoir et de connaissance, elle est aussi, profondément, affaire de discours, de travail sur le langage.

V. et I. : Justement, dans votre travail critique, n'y avait-il pas, à l'origine, une sorte de tension entre théorie et écriture, alors que dans les années récentes, vous insistez plutôt sur l'opposition entre recherche et création? Au lieu d'être une dialectique, la critique, dans le contexte actuel, serait devenue le choix d'un pôle par rapport à l'autre?

A. B.: Oui, il y a sans doute quelque chose de cela. D'emblée, je voyais la critique littéraire comme effectivement traversée par cette 
tension entre une orientation référentielle qui nous porte vers tout un monde d'idées et de significations, et l'écriture qui, elle, a davantage à voir avec la suggestion et qui comporte une part de création et d'expression. Je subordonnais très nettement cette dimension de l'expression à la dimension référentielle, mais quand même, pour moi, on ne pouvait pas arriver à réaliser la synthèse du savoir sur le texte, sur l'œuvre, sans l'intuition, c'est-à-dire sans quelque chose qui passait par les mots, par le langage, par l'écriture. Cela dit, plus tard, en effet, j'ai perçu la critique littéraire de plus en plus comme une affaire d'écriture. En tout cas, cet aspect de l'écriture est devenu pour moi de plus en plus important dans la mesure où se sont développées des approches du texte littéraire qui, elles, faisaient abstraction de cette dimension-là et qui correspondent en gros à ce que l'on peut appeler la "recherche". Une recherche qui est fondée soit sur l'érudition, soit encore sur la théorie, et qui n'est plus du tout intéressée à rendre compte de la spécificité du texte littéraire. Pour moi, l'intention de rendre compte de cette spécificité fait l'essentiel de la critique et rend obligatoire le recours à une "poésie d'idées", qui est une façon de soumettre le discours intellectuel à une intention, à une finalité axée sur la vérité de l'écriture.

V. et I. : La nouvelle critique est une question qui se posait à votre génération, du moins pour quelqu'un qui s'intéressait à la littérature. Même chose pour ce qui est des revendications de Parti pris, sans doute. Ce qui me frappe, par ailleurs, c'est qu'il y a une certaine distance entre vous et votre génération à d'autres points de vue. Par exemple, pour ce qui est de la contre-culture, qui a beaucoup sollicité les gens de votre âge. J'ai parfois le sentiment que c'est avec la génération précédente que vous cherchez davantage à dialoguer, comme vous l'avez d'ailleurs fait dans La Littérature et le Reste.

A. B.: Je pense que le phénomène de la génération est un phénomène très complexe et que l'on n'appartient jamais intégralement à une génération qui aurait tels caractères précis. On a toujours des liens avec la génération qui nous précède ou parfois avec celle qui nous suit. Certains intellectuels ou artistes sont portés à précéder en quelque sorte des phénomènes culturels, ils les annoncent longtemps d'avance; d'autres sont plutôt portés à rester en continuité avec tout un acquis. J'appartiens effectivement à ce qu'on peut appeler la génération de Parti pris, mais cette génération s'est, d'une certaine façon, dispersée assez rapidement, puisque quelqu'un comme Paul Chamberland, par exemple, qui était bel et bien de Parti pris, s'est retrouvé, lui, dans la contre-culture, donc avec de plus jeunes que lui. Sans doute, la génération de Parti pris et celle de la contre-culture coïnci- 
dent-elles en partie; mais les gens de Parti pris ne sont pas tous allés du côté de la contre-culture, loin de là. Même chose pour le marxisme. Jean-Marc Piotte, dans les années soixante-dix, était tout à fait au centre de la mouvance marxiste. Moi je ne l'étais pas, Paul Chamberland ne l'était plus, André Major ne l'a jamais été. Nous faisions quand même tous partie, à l'origine, d'un même groupe. J'étais très fier d'appartenir à une génération qui avait des idées nouvelles sur un tas de choses. Mais Michel van Schendel, qui avait une formation européenne et qui était marxiste, m'avait dit avec raison, peu de temps après le lancement de la revue, que c'était très superficiel, cette appartenance à une génération, et qu'il fallait bien plutôt concevoir une continuité entre les générations, si l'on voulait arriver à faire triompher certaines causes comme la cause socialiste, par exemple. Le Parti communiste en France n'était pas constitué d'une génération, mais de plusieurs générations qui reprenaient le même idéal et qui le faisaient progresser.

V. et I. : Évidemment, cette perspective de continuité n'était pas du tout celle des gens de Parti pris.

A. B. : Sur le plan culturel, certainement pas.

V. et I.: C'était tout de même la vôtre, notamment dans votre manière d'aborder la littérature en retournant au passé.

A. B.: Oui. Plusieurs autour de moi voulaient que la littérature québécoise commence avec Parti pris, qui avait discrédité avec raison l'appellation de littérature "canadienne-française". Moi, j'avais affirmé dès ce moment-là l'importance de montrer la continuité entre l'effort qui était le nôtre et celui des générations antérieures. Mais ce que j'allais repêcher dans la tradition littéraire, ce n'était pas l'intégralité des choses, comme on est porté à le faire aujourd'hui lorsqu'on réédite par exemple tout ce qui a pu s'écrire de textes versifiés au dixneuvième siècle. Dans mon esprit, il fallait agir de façon sélective, aller chercher dans le passé l'expression de ces forces vives qui pouvaient continuer d'alimenter le présent. Il n'y avait donc pas de rupture chez moi avec le passé, je suis bien d'accord avec vous. De la même façon, je n'étais pas un partisan de la généralisation du joual dans la littérature. J'ai sans doute eu dès le début des tendances qui me tournaient vers le passé. Ce n'était pas, je crois, du passéisme ou de la nostalgie, mais le besoin de constituer une littérature sur des bases solides. D'autre part, il y avait chez moi des aspects résolument novateurs sur le plan méthodologique, dans le recours à la critique thématique la plus inventive: celle de Poulet, Richard, Starobinski. J'ai d'ailleurs constitué ma propre méthode d'analyse des textes. J'entretenais 
un grand projet que je n'ai pas pu réaliser, peut-être parce que je n'avais pas suffisamment de foi - ça demande beaucoup de foi dans cette littérature qui "se faisait", pour reprendre l'expression de Gilles Marcotte, mais qui était loin d'être déjà réalisée. Mon grand projet aurait consisté à voir depuis le début de la littérature québécoise, depuis mettons François-Xavier Garneau et Crémazie en poésie, depuis au moins Laure Conan dans le domaine du roman, et en venant jusqu'à nous, comment une thématique québécoise se constituait, s'enrichissait peu à peu. Malheureusement, je n'ai eu ni le temps ni le courage de réaliser cela. Je l'ai fait de façon partielle bien sûr, en montrant à propos de tel auteur quels pouvaient être ses liens avec certaines ouvres antérieures, mais je rêvais quand même d'une espèce de vaste synthèse.

V. et I. : Après ce projet-là, qui était en partie réalisé dans L'Instance critique, j'ai cru comprendre qu'il vous a semblé plus urgent, plus important, de développer, au Québec, la critique de la littérature française?

A. B. : En effet. Je n'ai pas voulu me spécialiser dans la littérature québécoise. Ça me semblait très important de voir comment nous, Québécois qui nous nous nourrissions de littérature française, nous étions capables de nous approprier cette littérature, capables donc de la redéfinir et de l'ajuster à nos propres fins. C'était un très beau projet, mais encore moins réalisable que le précédent à cause de ses dimensions. En fait, ce qui m'a toujours intéressé, c'est une comparaison entre ce qu'on pourrait appeler la matrice littéraire québécoise et la matrice littéraire française, c'est-à-dire une sorte de disposition dynamique commune aux textes de chaque série. Mais cette comparaison à laquelle je voulais en arriver lorsque j'étais jeune et plein d'idéal, c'est extrêmement difficile à faire. Cela suppose qu'on ait traversé les deux traditions littéraires et qu'on ait dégagé à partir de là ce qui constitue cette matrice, cette épistémè, et qu'on puisse ensuite les mettre en comparaison. Ça aurait demandé en plus, de jeter un regard assez précis sur ce qui se fait aux États-Unis par exemple, parce qu'il y a dans la façon de concevoir le texte ici des éléments assez proches de ce qu'on peut trouver dans la littérature américaine. Quelques critiques comme Renald Bérubé et Jean-François Chassay s'y sont intéressés, mais il faudra beaucoup de temps et d'efforts, évidemment, pour arriver à une synthèse.

V. et I. : La valorisation de la littérature française se fait souvent, ces dernières années, par opposition à la littérature québécoise, et vice versa. Cela paraît résolument étranger à votre perspective depuis le début. 
A. B.: Oui. D'ailleurs, en 1968, dans une conférence sur l'enseignement de la littérature au Québec, j'avais bien dit que, pour moi, il n'était pas question d'un enseignement de la littérature québécoise qui se ferait sans lien avec la littérature française. Pour moi, il fallait enseigner les deux, et le travail que l'on pouvait faire sur l'une des littératures se trouvait à nourrir l'approche de l'autre. Cette perspective a été généralement abandonnée pendant les années soixante-dix et c'est là un des grands reculs de l'enseignement de la littérature au Québec. On 'en est venu à n'enseigner presque plus que la littérature québécoise, pendant cette décennie. Après le référendum de 1980, il y a eu le retour du balancier et on est revenu à la littérature française, mais souvent au détriment de la littérature québécoise. Il semble que beaucoup d'enseignants soient incapables d'assumer simultanément ces deux composantes essentielles de notre situation culturelle. La littérature française peut effectivement être perçue comme aliénante si on ne fait pas l'effort de se l'approprier et, pour se l'approprier, il faut développer des concepts, une approche, au contact de nos ouvres à nous.

V. et I.: Ces dernières années, vous avez surtout publié des récits et des recueils de poèmes. Jusqu'à quel point la critique fait-elle encore partie de vos projets?

A. B. : J'ai des projets de critique en relation avec mon enseignement. Par exemple, j'ai fait un cours sur Michel Tremblay. J'ai publié un article d'une quinzaine de pages sur certains aspects de son œuvre qui m'avaient particulièrement intéressé. Mais il y aurait de la matière pour une étude beaucoup plus longue, que j'écrirai peut-être un jour. J'ai aussi fait un cours intitulé "Roman et énumération", sur cinq auteurs français (Flaubert, Huysmans, Roussel, Yourcenar et Perec). Là encore, il s'agit d'une étude d'une assez grande étendue que je voudrais pousser jusqu'au bout. Le travail est déjà très avancé.

V. et I. : Il vous arrive souvent de fonctionner comme cela, c'est-à-dire en relation avec un cours?

A. B. : Oui, l'enseignement a toujours été pour moi l'occasion de préparer soit un article, soit même un livre. Le Langevin, par exemple, a été fait à partir d'un cours.

V. et I. : En ce qui concerne votre projet portant sur "Roman et énumération ", n'avez-vous pas le sentiment qu'écrire au Québec sur la littérature française est une entreprise difficile?

A. B. : Il est assez difficile, au Québec, de consacrer des monographies relativement considérables à des auteurs; déjà à des auteurs québé- 
cois, mais encore plus à des auteurs français. On a l'impression que ça ne va rejoindre qu'un public de cinquante personnes au plus. Cela m'a freiné souvent, et continue de me freiner lorsque j'envisage certains travaux de critique littéraire. Il y a aussi le fait que la critique littéraire est beaucoup moins en demande qu'il y a vingt ou trente ans. Maintenant, il semble qu'on ait pris de nouveau beaucoup de distance à l'égard des textes eux-mêmes, qu'on s'intéresse davantage à des phénomènes concernant le discours littéraire en général, ses implications sociales, etc. Ce qui m'importe personnellement, c'est ce que chaque texte apporte de particulier, non ce par quoi il s'insère dans un contexte. C'est, en somme, ce que chaque texte comporte d'ouverture au sens, à l'infini du sens. Chaque texte a une façon bien à lui de dire l'infini et c'est ça qui me passionne.

V. et I. : Dans La Vie aux trousses, n'est-ce pas d'ailleurs précisément cette perspective de l'، infini " qui structure le roman?

A. B. : Oui, elle est clairement thématisée dans La Vie aux trousses, qui comporte une part autobiographique. Il y a, au cœur même de l'existence du personnage, cette aspiration vers quelque chose de démesuré qui ne se définit jamais tout à fait, mais qui va être suggéré avec une assez grande précision tout de même, dans une expérience comme celle de la folie, ou disons du délire.

V. et I.: On observe souvent, me semble-t-il, une correspondance assez claire entre infini et délire dans vos romans.

A. B.: Le délire est certainement un accès à cet infini auquel chacun aspire, mais un accès désordonné. Je n'aime pas qualifier en termes positifs le délire, parce qu'il est totalement négatif à plusieurs égards. Mais en même temps, il peut donner accès à beaucoup de choses. Je pense à Nerval qui était un écrivain assez médiocre avant de faire l'expérience de la folie et qui, l'ayant connue, est devenu un grand écrivain. Cela ne veut pas dire que son écriture ne fait qu'un avec la folie, puisqu'il ne peut pas écrire au moment même où il la vit; mais la connaissance intime de la folie a décuplé, en quelque sorte, ses moyens.

V. et I. : Vous évoquiez à l'instant la part autobiographique de La Vie aux trousses. Cette part n'est-elle pas considérable dans l'ensemble de vos écrits?

A. B. : L'autobiographie est un concept assez complexe: on peut écrire dix ou vingt autobiographies différentes, toutes valables. J'ai souvent l'impression d'être dans le discours autobiographique et, en même temps, d'être tout à fait tourné vers autre chose, puisque l'autobiogra- 
phie, chez moi, c'est toujours quelque chose qui s'invente et qui n'a donc pas de rapport étroit avec la véracité.

V. et I.: Dans vos écrits de fiction, il me semble tout de même que vous n'allez pas volontiers vers des sujets qui seraient tout à fait étrangers à votre expériencè.

A. B. : Sans doute, mais j'ai l'impression que cela sera davantage possible pour moi dans mes prochains écrits. En tout cas, je fais un effort pour aller vers des sujets qui sont plus étrangers à moi. Mais au départ, en effet, il y a cela. Et c'est d'ailleurs la raison pour laquelle ce fut si long avant que j'arrive à l'écriture. Il m̀'a fallu beaucoup de temps avant de pouvoir assumer cette obligation où j'étais de passer par le moi. Je suis très introverti au départ - c'est un trait de caractère dont on est doté sans pouvoir rien y faire. Quand on est pris avec ça, il s'agit d'essayer d'en tirer quelque chose. On prend son parti d'être soi, si je puis dire, et alors on essaie d'exploiter cela de façon à rejoindre une vérité plus vaste. Je me dis qu'il y a beaucoup de gens qui sont aux prises eux aussi avec une lourde intériorité et qui, lisant ce que j'écris, pourront y retrouver peut-être quelque chose d'euxmêmes, pour la bonne raison que les moi sont constitués d'éléments qui ont quelque chose d'universel.

V. et I. : Vous voyez tout de même cela comme une perspective transitoire, de la même manière que vous voyiez la critique auparavant?

A. B.: Il s'agit pour moi de faire advenir quelque chose dans l'ordre de la connaissance de l'humain à partir de ce que je peux éprouver. L'un des buts que je me suis donnés en exploitant, mais à fond, cette dimension autobiographique, c'est précisément d'en arriver à dire ce que, en général, on ne dit pas, de façon à conquérir pour la connaissance littéraire des régions peu explorées, voire inexplorées. J'ai toujours été, par exemple, très conscient de certaines conduites de mauvaise foi qu'il peut y avoir chez moi comme chez tant d'autres, mais que personne n'avoue, évidemment. J'ai toujours été porté à mettre l'accent précisément sur ces aspects qui font que chacun est de mauvaise foi et qui gagnent par conséquent à être décrits, avoués, dévoilés. De là ce ton qu'on a pu qualifier de masochiste. Par exemple, dans La Croix du nord, il y a sans doute du masochisme. Mais il est assumé et utilisé à des fins de prospection littéraire. Il s'agit essentiellement pour moi d'élargir un peu le cercle des vérités concernant la réalité intérieure. On cache des démons, mais ces démons-là, je pense qu'on' a tout intérêt à les faire sortir, à leur faire prendre l'air. Par ailleurs, j'ai écrit récemment un roman dont le personnage principal n'est d'aucune façon une projection de moi-même. C'est quelqu'un de bien 
sympathique, un jeune, sans aucune parenté avec moi et j'ai réussi à mener ça jusqu'au bout.

V. et I. : Ce serait la première fois où vous auriez maintenu une véritable distance avec le protagoniste?

A. B.: Oui. J'espère bien continuer en ce sens. Il y a tout de même un problème, parce que pour aborder des sujets extérieurs à soi, il faut se renseigner, préciser sa connaissance de chaque chose, et je ne suis pas tellement porté vers ce genre de travail. La documentation, l'érudition représentent pour moi des corvées quasi insurmontables, sauf si elles sont orientées vers des points très précis qui m'intéressent.

V. et I. : Dans vos études critiques, les distinctions sont assez marquées entre les genres. Dans Le Singulier pluriel, par exemple, on voit que les genres constituent des blocs relativement distincts. Du côté de l'écriture proprement dite, comment voyez-vous cette différence entre les genres, particulièrement la poésie et les genres narratifs?

A. B. : La poésie, je la vois comme étant intimement traversée par un mouvement de prose. Il faut qu'il y ait quelque chose de très lié dans la poésie, même si la poésie se réalise à travers des images qui ont leur autonomie en quelque sorte et qui se développent pour ellesmêmes. Il faut un mouvement qui traverse le poème et ce mouvement-là, je le vois comme étant le fait d'une expressivité et, par conséquent, comme relevant de la prose. Je pense que prose et expressivité vont de pair alors que l'image, avec toute sa charge sémantique, relèverait plutôt de la fonction poétique dont parlait Jakobson. Lorsque j'écris en prose, j'essaie de développer une dimension narrative et je le fais beaucoup plus en rapport avec des réalités intérieures qu'avec des réalités extérieures. Je ne nie pas l'action extérieure, mais c'est avant tout une action intérieure qui se trouve mise en représentation. Aussi est-il essentiel pour moi que le langage accède à une certaine forme de poésie (je ne dirais pas de lyrisme, même si ça peut devenir du lyrisme à l'occasion), que le langage accède à une qualité de poésie, c'est-à-dire de suggestion. De là, je pense, le style assez effervescent de mes écrits. J'aime que la phrase puisse signifier à plusieurs niveaux. Il y a un sens immédiat qui doit être donné de façon très nette, mais en même temps, il faut qu'il y ait de la connotation.

V. et I. : Si bien que poésie et prose ne sont pas des terrains vraiment séparés?

A. B. : Sûrement pas. Pas dans mon cas, parce qu'il est bien sûr que mes récits, qui ont quelque chose d'autobiographique, qui développent beaucoup de situations à caractère intérieur, à caractère affectif 
marqué, ces récits-là ne peuvent être lus comme des romans d'aventures. Ils doivent être lus un peu comme des poèmes, mais des poèmes qui bougent...

V. et I. : Comment en êtes-vous venu à accorder autant de place, dans vos activités, à l'écriture poétique et narrative?

A. B.: Il y a des circonstances précises. Vous me pardonnerez d'en faire état un peu crûment. Pendant une dizaine d'années, j'ai eu plusieurs épisodes psychotiques, et puis j'ai été 'guéri par le lithium, un médicament tout à fait merveilleux. C'est à partir de là que j'ai pu enfin écrire. Mes tout premiers écrits, quand j'avais vingt ans, qu'il s'agisse de poésie ou de prose, étaient très contraints, très peu développés. Le véritable point de départ c'est en 1988, au moment où j'ai pris la décision d'écrire une heure par jour. Du jour au lendemain, je me suis mis à produire. Ça a été très soudain. Je me suis mis à écrire de la poésie, puis des récits. Ça s'est enchaîné. C'est le fait d'avoir dominé, maîtrisé la psychose par le lithium qui m’a permis cela. Jusque-là, je n'avais pas accès à mon imaginaire. Le propre du psychotique, me semble-t-il, c'est de ne pas avoir accès à l'imaginaire; il fonctionne uniquement au niveau symbolique. La guérison m'a donné la possibilité de me sentir de plain-pied dans l'écriture. Par èxemple, je peux maintenant imaginer une situation romanesque sans la remettre en question tout de suite, je peux faire comme si elle existait vraiment. Même chose pour la poésie: je peux aligner des mots et des images en ayant tout de suite le sentiment de leur pertinence. À croire que le doute - je veux dire : le doute qui paralyse - serait une disposition bassement physiologique...

V. et I. : Ce problème ne se posait pas pour la critique littéraire?

A. B. : Non, parce que le texte dont on parle est une donnée irrécusable, il existe en dehors du critique, et à ce moment-là, il s'agit simplement de constituer un appareil de notions qui, greffées au texte, vont le refléter, en rendre compte. L'existence est, pour ainsi dire, donnée par le texte. Le discours critique se constitue à partir d'elle. C'est ainsi qu'il arrive lui-même à exister d'une existence seconde, en quelque sorte. Bref, on peut être critique en étant psychotique, mais on peut difficilement être créateur sans avoir triomphé de la maladie. Voilà pourquoi il m'a fallu tant d'années pour accéder à l'écriture pleinement littéraire.

V. et I. : Comment voyez-vous aujourd'hui les rapports entre la littérature et la politique? Vous citez souvent ce vers de Roland Giguère: "une fête de fleurs sur une banquise perdue ". Cette banquise, dans $L a$ Grande Langue, semble décidément vous exaspérer. 
A. B.: Il m'a semblé que depuis quelque temps tous les principaux acquis de la Révolution tranquille étaient sabrés l'un après l'autre et que les Québécois, qui avaient voulu se comporter comme une majorité au Québec, acceptent graduellement de redevenir cette minorité à l'intérieur du Canada que nous étions avant la Révolution tranquille. Je' trouve cela extrêmement pénible. Il me semble que la loi 86 est très symptomatique d'un grand reniement de ce projet d'existence collective qui a été le nôtre depuis les années soixante. Qu'on accepte de redevenir bilingue (parce que c'est bien le but avoué de Claude Ryan avec la loi 86: refaire du Québec une société bilingue), cela m'a complètement dépassé. Je me suis dit: "Une fois de plus!" — parce que ces coups qui sont portés à notre idéal national se multiplient depuis dix ou quinze ans. Je me suis dit que nous étions une fois de plus ramenés à zéro. C'est tout à fait le mythe de Sisyphe... Mais là, vraiment, j'étais atterré, et le moyen pour moi de sortir du marasme, ce fut d'écrire, d'essayer d'extérioriser tous les affects profonds qui étaient touchés par cette mesure de la loi 86. La Grande Langue est née de là.

V. et I. : Cette longue antiphrase ne débouche-t-elle pas sur un profond découragement?

A. B. : Je voulais faire prendre conscience au lecteur de la gravité de la situation. Soutenir jusqu'au bout cette antiphrase - qui consiste, à peu près, à dire: "Tout est noir, réjouissons-nous, cédons joyeusement à l'assimilation" - il me semble que ça allait de soi. Ne jamais succomber, donc, à la tentation de se dire: "Dans le fond, ce n'est pas si grave", qui nous a fait avaler tant de couleuvres, depuis toujours.

V. et I. : Ni à la tentation de se dire que ça pourrait changer...

A. B.: Au moment où j'ai écrit La Grande Langue, il faut dire que j'étais vraiment désespéré. Maintenant, je voudrais bien être moins pessimiste. En tout cas, je suis certainement plus dégagé par rapport à cette question-là. Heureusement. On ne peut pas vivre en pensant comme ça que le Québec se meurt, que le Québec est mort. Je voudrais être moins pessimiste, mais il n'y a pas grand-chose dans l'actualité qui permette de penser qu'on va s'en sortir. Je suis très inquiet quand je pense à la dénatalité, à l'immigration massive. Il n'y a pas moyen d'intégrer des immigrants s'ils refusent d'apprendre le français et s'ils sont cinq ou dix fois trop nombreux. Il est donc facile d'imaginer que le Québec puisse rapidement être assimilé, lui qui ne se comporte plus comme une majorité.

V. et I. : Ces aspects paraissent relativement étrangers à la littérature. Jusqu'à quel point y a-t-il un lien à faire entre ce qui se passe du côté de la littérature et cette situation? 
A. B.: Quand j'ai commencé à écrire dans les années soixante, j'étais persuadé que si nous arrivions, tous ceux de ma génération et moi, à créer une littérature québécoise dynamique, vivante, cela voudrait dire que la partie était gagnée, que le peuple québécois existerait une fois pour toutes. Autrement dit, nous voyions un lien très immédiat entre la littérature et le Québec, le peuple. On peut se rendre compte aujourd'hui qu'on a parcouru beaucoup de chemin du côté de la littérature: beaucoup d'œuvres intéressantes, de grande qualité, ont paru et continuent à paraître. C'est très effervescent, encore, la littérature québécoise. Mais on a l'impression que ça ne veut plus du tout signifier que le Québec va choisir de rester français.

V. et I. : Il y a autrement dit comme une sorte de monde tout à fait parallèle.

A. B.: Oui. De là le recours à cette image de Roland Giguère: "une fête de fleurs sur une banquise perdue. Il me semble que dès cette époque, Giguère sentait quelque chose comme ça, c'est-à-dire le fait que, entre ce qu'on peut appeler, comme les marxistes, la superstructure et l'infrastructure, entre la culture et le groupe économique et politique, il n'y a pas nécessairement de continuité. J'espère bien que je me trompe. Finalement, tout est gagné si nous faisons l'indépendance. On peut penser qu'elle pourrait se faire assez rapidement, mais on peut aussi penser que, une fois de plus, ça va rater et, une fois de plus, il se sera perdu énormément d'efforts et d'énergie à essayer en vain de concevoir une structure politique viable. 